\title{
Exact solution and correlations of a dimer model on the checkerboard lattice
}

\author{
Julia Wildeboer $\odot,{ }^{1,2}$ Zohar Nussinov, ${ }^{3}$ and Alexander Seidel ${ }^{3}$ \\ ${ }^{1}$ Max-Planck-Institute for the Physics of Complex Systems, 01187 Dresden, Germany \\ ${ }^{2}$ Department of Physics, Arizona State University, Tempe, Arizona 85287-1504, USA \\ ${ }^{3}$ Department of Physics, Washington University, St. Louis, Missouri 63130, USA
}

(Received 19 April 2020; revised 11 June 2020; accepted 15 June 2020; published 6 July 2020)

\begin{abstract}
We present analytic results for a special dimer model on the nonbipartite and nonplanar checkerboard lattice that does not allow for parallel dimers surrounding diagonal links. We exactly calculate the number of closed packed dimer coverings on finite checkerboard lattices under periodic boundary conditions and determine all dimer-dimer correlations. The latter are found to vanish beyond a certain distance. We find that this solvable model, despite being nonplanar, is in close kinship with well-known paradigm-setting planar counterparts that allow exact mappings to $\mathbb{Z}_{2}$ lattice gauge theory.
\end{abstract}

DOI: 10.1103/PhysRevB.102.020401

\section{INTRODUCTION}

A major challenge in condensed matter theory lies in the endeavor of finding relatively simple toy models that are tractable and at the same time capture relevant universal physics. One long standing example for this is magnetic systems whose collective behavior can often be well described by relying on simple Ising- or Heisenberg-type models. Even the latter are, however, beyond analytic treatment in all but the simplest possible settings. The need for solvable models is particularly acute when investigating physics outside established paradigms.

Historically, models with dimer degrees of freedom (and their associated constraints) have played a key role in constructing solvable models in statical physics and, somewhat more recently, quantum magnetism. The enormous impact dimer models have had on various areas of theoretical physics can be traced back to Kasteleyn's observation that a large class of classical dimer models is solvable by Pfaffian methods on planar lattice graphs [1], followed by developments by Fisher [2] and Fisher and Stephenson [3], revealing deep connections with Ising models. This method has since been adopted to shine light on the phase diagram of quantum magnets. Kivelson, Rokhsar, and Sethna [4,5] have introduced the idea that quantum dimer models (QDMs) are effective descriptions of highly frustrated quantum magnets and can be tuned such that their ground-state correlations correspond to those of a classical dimer model [5]. This, in particular, is linked to scenarios of unconventional magnetism conceived during the advent of high-temperature superconductors [4,6-8]. In a seminal work, Moessner and Sondhi [9] demon-

Published by the American Physical Society under the terms of the Creative Commons Attribution 4.0 International license. Further distribution of this work must maintain attribution to the author(s) and the published article's title, journal citation, and DOI. Open access publication funded by the Max Planck Society. strated that Anderson's idea of a short-ranged resonating valence bond spin-liquid phase can be realized, under the assumption of validity of the spin-to-quantum-dimer mapping, on the triangular lattice. This assumption has been corroborated via a multitude of different approaches, including systematic expansion in an overlap parameter [10], which rests on the linear independence [11,12] of spin singlet (valence bond) states and the construction of $S U$ (2)-invariant spin-1/2 models that realize the same ground states $[13,14]$ and their physics [15-17]. Moreover, deep connections between certain QDMs and Kitaev's toric code for topological quantum computing [18] have long been appreciated, and the relation to the underlying Ising $\left(\mathbb{Z}_{2}\right)$ gauge theory can be made exact in the kagome lattice model discussed by Misguich et al. [19]. Other gauge theories describing QDMs on different lattices have been introduced and studied [8,20,21]. QDM type physics also appears in various orbital and spin-orbital systems [22] and Josephson junction arrays [23].

Up until now, the construction of dimer models has thus proven a profound and versatile tool, whose utility in the applications discussed above was, however, largely limited to planar lattice graphs. In this work, we show that no such limitation fundamentally exists. That is, we construct a dimer model on the checkerboard lattice, which is nonplanar due to crossing links, and has all of the benefits discussed above. In this work, we demonstrate the applicability of Pfaffian methods to this model. The model further allows exact mapping to Ising gauge theory, along with existences of pertinent local lattice symmetries, on which we will elaborate elsewhere.

\section{A NONPLANAR DIMER MODEL}

We will now introduce a dimer model acting on the space of restricted dimer coverings on the checkerboard lattice (Fig. 1). The model can be interpreted as a classical dimer model at infinite temperature, but at the same time, the correlations to be discussed have equal relevance to the ground state of a suitable QDM. The configuration space of this model is that of all possible dimer coverings subject to an additional 
(a)

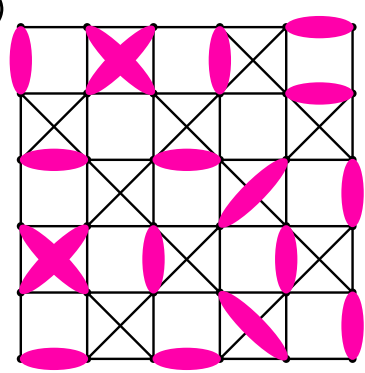

(b)

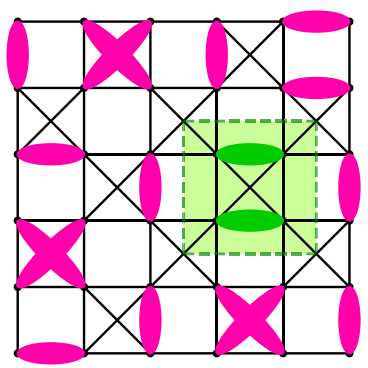

FIG. 1. The checkerboard lattice with some dimer coverings. (a) exemplifies a dimer covering that satisfies all constraints of our model. (b) A dimer covering that features a pair of parallel dimers (shaded) on the same crossed plaquette and is thus not allowed.

constraint. A "dimer covering" refers to a placing of dimers on some of the links of a lattice, such that each vertex belongs to exactly one such dimer. Here, crossed dimers on crossedlinked plaquettes are explicitly allowed. However, we impose the restriction that cross-linked plaquettes may not admit a pair of parallel dimers (Fig. 1 and caption). Effectively, we thus introduce an interaction ascribing a very large energy to pairs of dimers occupying the vertical or horizontal links on a cross-linked plaquette, whereas there is no such penalty for single or double occupation of crossed links. In the following, we will set this energy penalty to infinity first and then consider the infinite temperature partition sum of the resulting constrained dimer model.

It is worth pointing out that constrained dimer models on nonplanar lattices have appeared in the literature before [24], though typically, the constraint disallows crossed dimers. Even then, the nonplanarity of the model is evident by the fact that typical overlap graphs [25] between two different dimer coverings will have many crossings. We are not aware, however, of any such model that allows exact calculation of partition/correlation functions, which is what we will now turn to for the model at hand. To appreciate why that is possible, we first make contact with a well-known theorem by Kasteleyn [1], which, however, applies only to planar lattices.

According to this theorem, for any planar graph, an antisymmetric matrix $A$ can be found such that the infinite temperature dimer partition function is given by the Pfaffian (Pf) of the matrix $A$. This matrix encodes a link orientation of the graph, which has certain additional properties making it a "Kasteleyn orientation." Here, the indices of $A$ are site indices of the lattice, and $A_{i j} \neq 0$ only if $i, j$ are connected by a link. The orientation is defined by arrows placed along the links [Fig. 2(b)], and $A_{i j}=1$ if the arrow points from $i$ to $j, A_{i j}=-1$ if it points from $j$ to $i$. The defining property of a Kasteleyn orientation is to place arrows on the links of the planar lattice so that each plaquette is "clockwise odd," e.g., the number of clockwise arrows around any elementary plaquette (face) is odd. Reference [26] introduced the notion of a "pre-Kasteleyn" orientation. This notion is meaningful even for nonplanar two-dimensional lattices, i.e., lattices equipped with crossing links. An orientation for such links was defined to be "pre-Kasteleyn" if for any closed, non-self-intersecting, contractible loop along links, the number of clockwise oriented links is even if the number of sites enclosed by the loop

(a)

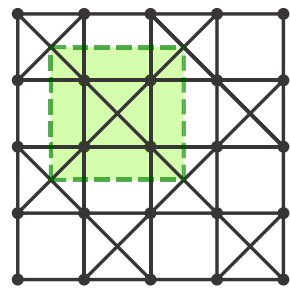

(b)

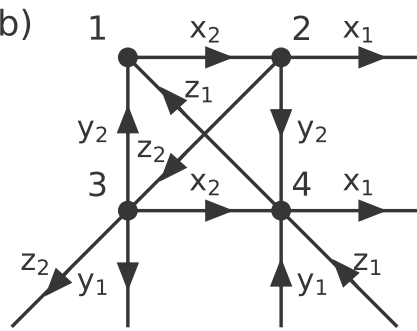

FIG. 2. (a) The checkerboard lattice is shown; the unit cell is depicted inside the dashed lines. (b) shows the four-site unit cell of the checkerboard lattice. It contains twelve links each equipped with a respective weight of $x_{1}, x_{2}, y_{1}, y_{2}, z_{1}, z_{2}$. The arrows on the links indicate the Kasteleyn orientation.

is odd, and vice versa. The term "contractible" is necessary only in the presence of nontrivial boundary conditions, in particular toroidal, periodic ones. The main difference between Kasteleyn and pre-Kasteleyn orientations is that for the latter, we do not need well-defined notions of an "elementary plaquette," or face, of the lattice. We only need the lattice to be meaningfully embedded within a two-dimensional planar or toroidal surface, so that "enclosed" is well defined. The two notions agree, however, for planar lattice graphs [26]. The arrows shown in Fig. 2(b) do endow the checkerboard lattice with a pre-Kasteleyn orientation. This follows from the fact that the checkerboard is obtained form the frustrated square lattice (with all square cross-linked) and its pre-Kasteleyn orientation discussed in Ref. [26] via removal of links.

For an ordinary Kasteleyn orientation, and assuming open boundary conditions, one has that $\operatorname{Pf}(A)$ equals (up to a sign) the number of all dimer coverings, i.e., the infinite temperature dimer partition function. Similarly, for our pre-Kasteleyn orientation, $\operatorname{Pf}(A)$ can be written as a sum, over dimer coverings, of terms \pm 1 . Here the positive (negative) sign corresponds to dimer coverings with an even (odd) number of crossed dimers. We now define "physical" dimer coverings as those that do not have parallel dimers on any cross-linked plaquette. On the checkerboard lattice, any unphysical dimer covering is uniquely associated to a physical one, by replacing all unphysical parallel pairs with crossed dimers on the same plaquette. If we now consider any pair of crossed dimers together with the two unphysical configurations associated to it, we find that these three local configurations contribute $-1+1+1=1$ to $\operatorname{Pf}(A)$. One then easily realizes that, for checkerboard lattices of any size and shape (open boundary conditions), $\operatorname{Pf}(A)$ gives the number of physical dimer configurations. Such counting problems certainly have great tradition in the field $[1,2]$. One has the intuition that whenever this counting is possible, then, at the very least, correlations can also be calculated. This will indeed turn out to be the case.

We proceed by reviewing nuts and bolts of Kasteleyn's formalism. Assuming, now, periodic boundary conditions, the partition sum for all dimer coverings is given by [27]

$$
Z=\frac{1}{2}\left(-\operatorname{Pf}\left(A^{00}\right)+\operatorname{Pf}\left(A^{\frac{1}{2} 0}\right)+\operatorname{Pf}\left(A^{0 \frac{1}{2}}\right)+\operatorname{Pf}\left(A^{\frac{1}{2} \frac{1}{2}}\right)\right),
$$

where $A^{00}$ encodes the (pre)-Kasteleyn orientation as before, in the presence of periodic boundary conditions. The other three matrices are the same, except for the presence of vertical 
and/or horizontal boundary "twists." Here, a twist introduces a flip of orientation along all links crossed by a closed path that traverses the lattice horizontally (vertically) as indicated by a $\frac{1}{2}$ in the first (second) index. The Pfaffians $\operatorname{Pf}\left(A^{\alpha \beta}\right)$ then satisfy

$$
\operatorname{Pf}\left(A^{\alpha \beta}\right)= \pm \sqrt{\operatorname{det}\left(A^{\alpha \beta}\right)}
$$

where we will fix the signs below. The matrices $A^{\alpha \beta}$ can be block diagonalized by a Fourier transformation, giving

$$
\operatorname{det}\left(A^{\alpha \beta}\right)=\prod_{n=0}^{N-1} \prod_{m=0}^{M-1} \operatorname{det} A\left(\theta_{n, \alpha}, \phi_{m, \beta}\right),
$$

where the matrix blocks $A\left(\theta_{n, \alpha}, \phi_{m, \beta}\right)$ are given by [28]

$$
A\left(\theta_{n, \alpha}, \phi_{m, \beta}\right)=\sum_{M_{1}, N_{1}} a_{M_{1}, N_{1}} e^{i\left(N_{1} \theta_{n, \alpha}+M_{1} \phi_{m, \beta}\right)} .
$$

Here, the phases $\theta_{n, \alpha}$ and $\phi_{m, \beta}$ are specified via

$$
\theta_{n, \alpha}=\frac{2 \pi(n+\alpha)}{N} \quad \text { and } \quad \theta_{m, \beta}=\frac{2 \pi(m+\beta)}{M}
$$

with $n=0, \ldots, N-1$ and $m=0, \ldots, M-1 . M(N)$ is the number of unit cells as depicted in Fig. 2(b) in vertical (horizontal) direction. The $a_{M_{1}, N_{1}}$ encode the Kasteleyn orientation as follows: Let $j=\left(M_{1}, N_{1}, v\right)$ be a multi-index specifying the lattice site in the unit cell given by indices $M_{1}, N_{1}$ and corresponding to a unit-cell basis-index $v$, and let similarly $i=(0,0, \mu)$ specify a lattice site in the $(0,0)$-unit cell, then $\left[a_{M_{1}, N_{1}}\right]_{\mu, v}=A_{i j}$. Formally, $M_{1}$ and $N_{1}$ also run over $M$ and $N$ distinct values, respectively, but only values $M_{1}, N_{1}=$ $-1,0,1$ will lead to nonzero $a_{M_{1}, N_{1}}$, referring to a unit cell $(0,0)$ and its neighbors.

The pre-Kasteleyn orientation of Fig. 2(b) does, by itself, not enlarge the two-site unit cell of the checkerboard lattice.
However, we may be interested in a more general problem by endowing links with certain positive weights $x_{k}, y_{k}, z_{k}$, $k=1,2$, as shown in the figure. The weights multiply the corresponding matrix elements of $A_{i j}$. For $x_{1}^{2}+y_{1}^{2} \geqslant z_{1} z_{2}$, $x_{2}^{2}+y_{2}^{2} \geqslant z_{1} z_{2}$ the identification of unphysical parallel dimer pairs with crossed pairs can still be interpreted as a positive partition function. The resulting unit cell then has four sites. Though in the end, we mostly will be interested in the case with all weights equal to 1 , it has certain advantages to think of the larger unit cell whose sites comprise one crossed plaquette, garnished with the weights shown in Fig. 2(b). In the following, we will refer to this unit cell, which contains twelve links.

The nine nonzero matrices $a_{M_{1}, N_{1}}$ may now be read off from Fig. 2(b). One has

$$
\begin{aligned}
& a_{0,1}=\left(\begin{array}{cccc}
0 & 0 & 0 & 0 \\
x_{1} & 0 & 0 & 0 \\
0 & 0 & 0 & 0 \\
0 & 0 & x_{1} & 0
\end{array}\right), \quad a_{1,0}=\left(\begin{array}{rrrr}
0 & 0 & 0 & 0 \\
0 & 0 & 0 & 0 \\
y_{1} & 0 & 0 & 0 \\
0 & -y_{1} & 0 & 0
\end{array}\right), \\
& a_{1,1}=\left(\begin{array}{rrrr}
0 & 0 & 0 & 0 \\
0 & 0 & 0 & 0 \\
0 & 0 & 0 & 0 \\
-z_{1} & 0 & 0 & 0
\end{array}\right), \quad a_{1,-1}=\left(\begin{array}{cccc}
0 & 0 & 0 & 0 \\
0 & 0 & 0 & 0 \\
0 & z_{2} & 0 & 0 \\
0 & 0 & 0 & 0
\end{array}\right) \text {, } \\
& a_{0,0}=\left(\begin{array}{rrrr}
0 & x_{2} & -y_{2} & -z_{1} \\
-x_{2} & 0 & z_{2} & y_{2} \\
y_{2} & -z_{2} & 0 & x_{2} \\
z_{1} & -y_{2} & -x_{2} & 0
\end{array}\right)
\end{aligned}
$$

Furthermore,

$$
\begin{aligned}
& a_{-1,0}=-a_{1,0}^{T}, \quad a_{0,-1}=-a_{0,1}^{T}, \\
& a_{-1,-1}=-a_{1,1}^{T}, \quad a_{-1,1}=-a_{1,-1}^{T} .
\end{aligned}
$$

The matrices $A\left(\theta_{n, \alpha}, \phi_{m, \beta}\right)$ can be written as

$$
\begin{aligned}
A\left(\theta_{n, \alpha}, \phi_{m, \beta}\right) & =\sum_{M_{1}, N_{1}} a_{M_{1}, N_{1}} e^{i\left(N_{1} \theta_{n \alpha}+M_{1} \phi_{m \beta}\right)} \\
& =a_{0,0}+a_{0,1} e^{i \theta_{n}}-a_{0,1}^{T} e^{-i \theta_{n}}+a_{1,0} e^{i \phi_{m}}-a_{1,0}^{T} e^{-i \phi_{m}}+a_{1,1} e^{i\left(\theta_{n}+\phi_{m}\right)}-a_{1,1}^{T} e^{-i\left(\theta_{n}+\phi_{m}\right)}+a_{-1,1} e^{-i\left(\theta_{n}-\phi_{m}\right)}-a_{-1,1}^{T} e^{i\left(\theta_{n}-\phi_{m}\right)} \\
& =\left(\begin{array}{cccc}
0 & x_{2}-x_{1} e^{-i \theta_{n}} & -y_{2}-y_{1} e^{-i \phi_{m}} & -z_{1}+z_{1} e^{-i\left(\theta_{n}+\phi_{m}\right)} \\
x_{2}+x_{1} e^{i \theta_{n}} & 0 & z_{2}-z_{2} e^{i\left(\theta_{n}-\phi_{m}\right)} & y_{2}+y_{1} e^{-i \phi_{m}} \\
y_{2}+y_{1} e^{i \phi_{m}} & -z_{2}+z_{2} e^{-i\left(\theta_{n}-\phi_{m}\right)} & 0 & x_{2}-x_{1} e^{-i \theta_{n}} \\
z_{1}-z_{1} e^{i\left(\theta_{n}+\phi_{m}\right)} & -y_{2}-y_{1} e^{i \phi_{m}} & -x_{2}+x_{1} e^{i \theta_{n}} & 0
\end{array}\right) .
\end{aligned}
$$

Any single block (8) is, in general, not skew symmetric and so by itself does not represent a well-defined contribution to the Pfaffian. However, such a block comes with a conjugate partner, and a change of basis within the two blocks (corresponding to a real, sine-cosine Fourier transform of the original matrix) restores skew symmetry and shows that such partners contribute a positive factor to the Pfaffian [29]. For even $M, N$, except in $A^{00}$, all blocks (8) come with conjugate partners, so the Pfaffians of the remaining three $A^{\alpha \beta}$ lead to a positive contribution. For $A^{00}$, the sign of the Pfaffian may be worked out from the four special, already skew symmetric blocks with $\theta, \phi \in\{0, \pi\}$. One then finds that it is given by the sign of

$$
\begin{aligned}
& {\left[\left(x_{1}+x_{2}\right)^{2}+\left(y_{1}+y_{2}\right)^{2}-4 z_{1} z_{2}\right]} \\
& \quad \times\left[\left(x_{1}-x_{2}\right)^{2}+\left(y_{1}-y_{2}\right)^{2}-4 z_{1} z_{2}\right],
\end{aligned}
$$

where the first factor is non-negative under our earlier assumption, $x_{1}^{2}+y_{1}^{2} \geqslant z_{1} z_{2}$. In particular, if we now specialize to $x_{1}=x_{2}=x, y_{1}=y_{2}=y$ and $z_{1}=z_{2}=z$ for simplicity, the sign of $\operatorname{Pf} A^{00}$ is negative, Eq. (1) becomes

$$
Z=\frac{1}{2} \sum_{\alpha \beta} \sqrt{\operatorname{det}\left(A^{\alpha \beta}\right)} \text { for } \alpha, \beta=0, \frac{1}{2} \text {. }
$$


From (8),

$$
\begin{aligned}
\operatorname{det} A\left(\theta_{n, \alpha}, \phi_{m, \beta}\right)= & 4\left[x^{2}+y^{2}+\left(z^{2}-x^{2}\right) \cos \theta\right. \\
& \left.+\left(y^{2}-z^{2}\right) \cos \phi\right]^{2} .
\end{aligned}
$$

In the thermodynamic limit, the distinction between "twists" $(\alpha, \beta)$ becomes irrelevant, and we may evaluate the per-dimer free energy as

$$
\begin{aligned}
f(x, y, z)= & \frac{1}{2(2 \pi)^{2}} \int_{0}^{2 \pi} \int_{0}^{2 \pi} \ln \operatorname{Pf}[A(\theta, \phi)] d \theta d \phi \\
= & \frac{1}{2 \pi} \int_{0}^{2 \pi} \log \left[x^{2}+y^{2}+\left(z^{2}-x^{2}\right) \cos \theta\right. \\
& \left.+\sqrt{\left(x^{2}+y^{2}+\left(z^{2}-x^{2}\right) \cos \theta\right)^{2}-\left(z^{2}-y^{2}\right)^{2}}\right] d \theta .
\end{aligned}
$$

It is interesting to evaluate this last expression in the limit $z^{2} \rightarrow x^{2}+y^{2}$, when the effective weight of crossed dimers becomes zero. In this limit we have a highly constrained model, where diagonal dimers are still possible, but no pair of dimers, parallel or crossed, may occupy a cross-linked plaquette. One may infer from the last equation that this is a critical point, where

$$
f=\frac{x^{2}+y^{2}-z^{2}}{2 \pi\left(y^{2}-z^{2}\right)^{2}} \log \left(x^{2}+y^{2}-z^{2}\right)+\ldots,
$$

and where the ellipses represent less singular terms. It is interesting to note that field-theoretic mappings indicate an abundance of (first-order) transitions in the phase diagram of the frustrated square lattice (with one diagonal in each square) [30]. The present model gives analytic access to similar transitions in a microscopic setting.

Specializing from now on to $x=y=z$, and going back to a finite lattice with PBCs, Eqs. (8)-(11) directly give

$$
Z=2 \cdot\left(4 x^{2}\right)^{M N} \stackrel{x=1}{=} 2 \cdot 4^{M N},
$$

where the $x=1$ result is the number of dimer coverings of the lattice of $M N$ (four-site) unit cells [31]. Interestingly, the latter is formally the same expression as for the kagome. We caution, however, that the number of sites per unit cell is different for the checkerboard and kagome, so the counting in terms of lattice sites is different.

\section{Correlations}

Though the present case represents a highly nonstandard application of Pfaffian methods, experience nonetheless suggests that if $Z$ is computable, then so are correlation functions. We will now show that this is indeed the case. We are interested in the correlation of the dimer operator $n_{i j}$, where $i$ and $j$ denote neighboring lattice sites, and $n_{i j}=1(0)$ if the link $i j$ is occupied (empty). Since products of these operators are projection operators, their expectation values can be written as $Z^{\prime} / Z$, where $Z$ is the original partition function, and $Z^{\prime}$ the partition function restricted to the subspace onto which the operator in question projects. In practice, $Z^{\prime}$ is the partition function of the same lattice with certain links removed. E.g., if $i j$ is a horizontal or vertical link, one may easily see that dimerizations that have this link occupied, subject to our no-double-occupancy rule, are in one-to-one correspondence with dimerizations of the same lattice that have all other links of the cross-linked square containing $i j$ removed, as well as all other links attaching to either $i$ or $j$. For a diagonal link $i j$, the same prescription effectively leads to counting all dimerizations having this link occupied but not crossed. Let's call the associated partition function $Z^{\prime \prime}$. The partition function $Z^{\prime \prime \prime}$ of configurations where $i j$ is occupied and crossed is similarly related to the partition function of the lattice with all links on or attached to the cross-linked plaquette removes, except for the cross [32]. Thus $Z^{\prime}=Z^{\prime \prime}+Z^{\prime \prime \prime}$. Products of $n_{i j}$ operators are dealt with accordingly. Based on these observations, the calculation of correlation functions for the present problem differs only slightly from the standard case of unrestricted dimer coverings of a planar lattice graph. The difference is only in working out the links to be removed for a given numerator $Z^{\prime}$. We may denote by $\Delta$ the matrix obtained from $A$ by keeping only those matrix elements corresponding to removed links, setting the others equal to zero. It is then standard to express $Z^{\prime} / Z$ (or $Z^{\prime \prime} / Z, Z^{\prime \prime \prime} / Z$ ) in terms of $\Delta$ and the Green's function matrix, $G=A^{-1}$. We review this technique in the Supplemental Material [33].

We are now interested in connected correlation functions

$$
C[i j, k l]=\left\langle n_{i j} n_{k l}\right\rangle-\left\langle n_{i j}\right\rangle\left\langle n_{k l}\right\rangle .
$$

Classical and quantum dimer models throughout the literature exhibit a great variety of behaviors, including power-law [34-36] and (super)-exponentially decaying $[9,19]$ correlations, mirrored by a class of closely related spin-degree wave functions $[19,37,38]$. In the present case, we find the correlations (15) to be ultra-short ranged, i.e., nonvanishing only up to a certain finite distance. The finitely many nonzero values of the correlator are listed in Tables I-III of the Supplemental Material [33]. This property is familiar from a few select dimer models, notably that on the kagome lattice [19]. It hints at a deeper solvable structure of the present model, which we now outline.

\section{DISCUSSION AND CONCLUSION}

Planar dimer models exhibit a plethora of phases, including broken symmetry and $\mathbb{Z}_{2}$-topological phases. The correlators addressed in the preceding section do not indicate any broken symmetry. On the other hand, with periodic boundary conditions, dimer coverings can be subdivided into four topological sectors, as familiar from planar dimer models [25]. The latter transform nontrivially under symmetries of the lattice. Related to this, any absence of symmetry breaking in dimer models has long been associated to topological order $[39,40]$. Indeed, these arguments may be sharpened when considering quantum dimer models of the RK type, whose ground state correlators agree with those of the classical model considered here. In particular, this then allows one to study question of universality through entanglement properties of the ground state, and properties of the excitations, such as braiding statistics. Such a program can be carried out in full detail for the present model. Here we summarize key features, while details will be given elsewhere [41].

It is worth noting that a small subset of QDMs are fully solvable-all eigenstates are known, not just the RK ground 
state. This is in particular true for the kagome QDM [19], which can be written as the sum of commuting local operators, permitting an exact mapping to Ising gauge theory, and for which the vanishing of all correlations between local operators at sufficient distance can be demonstrated exactly [19,42-44]. Despite its nonplanarity, we have noted a number of parallels between the present model and the kagome case. This is no coincidence. The key uniting feature between these models turns out to be the existence of an arrow representation for permissible dimer coverings, which has been appreciated for the kagome for some time [45]. This translates the construction principle for the kagome QDM to the present case, with all the benefits mentioned. Moreover, the calculation of ground state entanglement entropy is pos- sible exactly, exposing a topological part of $\ln 2$, proving the topological nature of the ground state. Finally, quasiparticle statistics are accessible through modular properties of socalled minimally entangled states (MES) [46], which are again exactly computable for the QDM associated to the present model.

The purpose of this work is to illustrate that a wealth of beautiful models realizing topological orders lies hidden in nonplanar dimer physics. Such models can be made accessible through the notion of a pre-Kasteleyn orientation. We have discussed a checkerboard model that is fully solvable and whose quantum version describes a topological liquid. We are hopeful that this approach will stimulate many fruitful developments.
[1] P. W. Kasteleyn, Physica 27, 1209 (1961).

[2] M. E. Fisher, Phys. Rev. 124, 1664 (1961).

[3] M. E. Fisher and J. Stephenson, Phys. Rev. 132, 1411 (1963).

[4] S. A. Kivelson, D. S. Rokhsar, and J. P. Sethna, Phys. Rev. B 35, 8865 (1987).

[5] D. S. Rokhsar and S. A. Kivelson, Phys. Rev. Lett. 61, 2376 (1988).

[6] P. W. Anderson, Science 235, 1196 (1987).

[7] P. Fazekas and P. W. Anderson, Philosophical Magazine 30, 423 (1974).

[8] E. Fradkin and S. Kivelson, Mod. Phys. Lett. B 04, 225 (1990).

[9] R. Moessner and S. L. Sondhi, Phys. Rev. Lett. 86, 1881 (2001).

[10] A. F. Albuquerque, D. Schwandt, B. Hetenyi, S. Capponi, M. Mambrini, and A. M. Läuchli, Phys. Rev. B 84, 024406 (2011)

[11] J. T. Chayes, L. Chayes, and S. A. Kivelson, Comm. Math. Phys. 123, 53 (1989).

[12] J. Wildeboer and A. Seidel, Phys. Rev. B 83, 184430 (2011).

[13] A. Seidel, Phys. Rev. B 80, 165131 (2009).

[14] B. Normand and Z. Nussinov, Phys. Rev. Lett. 112, 207202 (2014).

[15] J. Wildeboer and A. Seidel, Phys. Rev. Lett. 109, 147208 (2012).

[16] Z. Zhou, J. Wildeboer, and A. Seidel, Phys. Rev. B 89, 035123 (2014).

[17] J. Wildeboer, A. Seidel, and R. G. Melko, Phys. Rev. B 95, 100402(R) (2017).

[18] A. Y. Kitaev, Ann. Phys. 303, 2 (2003).

[19] G. Misguich, D. Serban, and V. Pasquier, Phys. Rev. Lett. 89, 137202 (2002).

[20] R. Moessner, S. L. Sondhi, and E. Fradkin, Phys. Rev. B 65, 024504 (2001).

[21] F. S. Nogueira and Z. Nussinov, Phys. Rev. B 80, 104413 (2009).

[22] F. Vernay, A. Ralko, F. Becca, and F. Mila, Phys. Rev. B 74, 054402 (2006).

[23] A. F. Albuquerque, H. G. Katzgraber, M. Troyer, and G. Blatter, Phys. Rev. B 78, 014503 (2008).

[24] H. Yao and S. A. Kivelson, Phys. Rev. Lett. 108, 247206 (2012).

[25] E. Fradkin, Field Theories of Condensed Matter Systems (Westview, Oxford, 1997).
[26] J. Wildeboer and A. Seidel, Phys. Rev. B 101, 224406 (2020).

[27] This statement carried over from the Kasteleyn orientation of the square lattice [1] to our checkerboard pre-Kasteleyn orientation.

[28] L. Breiman and E. Beckenbach, Applied Combinatorial Mathematics (Wiley, New York, 1964), pp. 284-319.

[29] The determinant overall matrix transformation is taken into account in all pertinent arguments.

[30] F. Trousselet, P. Pujol, F. Alet, and D. Poilblanc, Phys. Rev. E 76, 041125 (2007).

[31] One may similarly show that all four "topological sectors" in the presence of PBCs contribute equally to this number.

[32] A factor of $(-1)$ must be accounted for due to the presence of the cross.

[33] See Supplemental Material at http://link.aps.org/supplemental/ 10.1103/PhysRevB.102.020401 for further details on dimer expectation values and correlations. Short-distance connected correlations between all twelve links contained in the four-site unit cell of the dimer model on the checkerboard lattice are obtained.

[34] P. W. Leung, K. C. Chiu, and K. J. Runge, Phys. Rev. B 54, 12938 (1996).

[35] F. Alet, J. L. Jacobsen, G. Misguich, V. Pasquier, F. Mila, and M. Troyer, Phys. Rev. Lett. 94, 235702 (2005).

[36] D. A. Huse, W. Krauth, R. Moessner, and S. L. Sondhi, Phys. Rev. Lett. 91, 167004 (2003).

[37] S. Fujimoto, Phys. Rev. B 72, 024429 (2005).

[38] B. Sutherland, Phys. Rev. B 37, 3786 (1988).

[39] N. Read and B. Chakraborty, Phys. Rev. B 40, 7133 (1989).

[40] S. Kivelson, Phys. Rev. B 39, 259 (1989).

[41] J. Wildeboer, Z. Nussinov, and A. Seidel (unpublished).

[42] S. Furukawa, G. Misguich, and M. Oshikawa, Phys. Rev. Lett. 96, 047211 (2006).

[43] F. Wang and F. Y. Wu, Phys. Rev. E 75, 040105(R) (2007).

[44] F. Wu and F. Wang, Phys. A (Amsterdam, Neth.) 387, 4148 (2008).

[45] V. Elser, Phys. Rev. Lett. 62, 2405 (1989).

[46] Y. Zhang, T. Grover, A. Turner, M. Oshikawa, and A. Vishwanath, Phys. Rev. B 85, 235151 (2012). 